\title{
REVISIÓN DE LA ADAPTACIÓN DEL PICO EN ALGUNOS COLIBRIES AMAZÓNICOS (AVES, TROCHILIDAE) CON RESPECTO A LAS FLORES QUE VISITAN
}

\author{
Sheila Figueroa-Ramírez ${ }^{1}$ \\ V. Morales ${ }^{1}$
}

\begin{abstract}
RESUMEN
La estrecha correlación evolutiva entre las flores y los colibríes es evidente por las estructuras morfológicas del pico y las formas de corola que visitan. Se analizaron algunas medidas morfológicas de los cráneos de cinco especies de colibríes (Phaethornis superciliosus, P. bourcieri, Glaucis hirsuta, Threnetes leucurus y Thalurania furcata), procedentes de la Amazonía de Loreto, Perú, depositados en el Museo de Historia Natural de la URP. Se hizo el análisis de componente principal (ACP) para las medidas morfométricas de los cráneos y observar las correlaciones del cráneo y los hábitos ecológicos de las especies. Los picos de las especies Thalurania furcata, Threnetes leucurus, Glaucis hirsuta y Phaethornis bourcieri están fuertemente asociados a la Inserción del atlas (IA) y los picos de Phaethornis superciliosus por la distancia entre las Narinas $(\mathrm{Nr})$ y el ángulo de inserción del pico con respecto a la columna (AP).
\end{abstract}

Palabras claves: Morfometría craneana, Trochilidae, correlación, Loreto, Perú.

\section{SUMMARY}

The closest evolutionary correlation between flowers and hummingbirds is evident by the specific connection of the morphologic structures from the bill and flower's corolla that they visit. They were analyzed some morphologic measures of five species from hummingbird's skulls (Phaethornis superciliosus, P. bourcieri, Glaucis hirsuta, Threnetes leucurus y Thalurania furcata), from the

1 Museo de Historia Natural. Universidad Ricardo Palma. Av. Benavides 5440, Santiago de Surco, Lima - 33, Perú. Apartado Postal 18-01. 
Amazonia of Loreto, Peru, deposited in the Museum of Natural History from URP. This study makes the analysis of principal component (ACP) for the skull's morphologic measures and to observe the skull's correlations and the ecological habits of these species. The bills from the species Thalurania furcata, Threnetes leucurus, Glaucis hirsuta and Phaethornis bourcieri are strongly associated by the atlas insertion (IA) and the bills from Phaethornis superciliosus are associated by the distance between their narines $(\mathrm{Nr})$ and by the angle insertion of the bill respect to the column (AP).

Keywords: Morphologic measures, skulls, Trochilidae, correlation, Loreto, Peru.

\section{INTRODUCCIÓN}

El Pico es el órgano principal de las aves para atrapar, romper y tragar los alimentos. Esta estructura está formada por el hueso de ambas mandíbulas y cubierta por una funda córnea llamada ranfoteca que es lo que observamos externamente.

La forma del pico está determinada por el tipo de alimento que consume el ave y guarda correlación con el punto de gravedad de la columna.

Los colibríes están adaptados para alimentarse del néctar de flores y a la vez contribuyen a la polinización. Por otro lado, la presión competitiva intra e interespecifica es atenuada entre las especies e incluso entre sexos por variaciones en el comportamiento de forrajeo, en la preferencia de microhabitats y en el largo y curvatura del pico (Temeles y Kress 2003), facilitando la diferenciación y participación en el uso de los recursos florales.
Diferentes estudios han demostrado la relación que existe entre morfología y ecología. (Stiles 1995, Grant y Grant 2003, entre otros). Sin embargo, no se han realizado análisis ecomorfológicos que relacionen los colibríes y sus recursos. (Miles y Ricklefs 1984, Losos 1990, Losos et al 2003).

Las estructuras craneales de los colibríes están adaptadas para sostener la estructura del pico. El cráneo se ha adaptado al largo, ancho, inclinación del ángulo del pico, inserción del pico, etc., para hacer eficiente la alimentación y la especificidad del pico para cada flor nectária.

El presente trabajo busco identificar las relaciones predicativas entre pico cráneo y las diferentes flores nectárias que utilizan para su alimentación.

\section{MATERIAL Y MÉTODOS}

Se examinaron a las especies Phaethornis superciliosus, Phaethornis 
bourcieri, Thalurania furcata, Threnetes leucurus y Glaucis hirsuta, las cuales se obtuvieron del Museo de Historia Natural de la Universidad Ricardo Palma (Lima-Perú). Estas muestras fueron originalmente colectadas de en la Amazonía peruana (Loreto), la cual es una zona típica de bosque lluvioso tropical, la cual favorece a la vegetación de la cual se alimentan estas especies de colibríes.

Para el análisis de la relación picocráneo se tomaron las siguientes medidas: Largo del pico (LP), desde el proceso supra-orbital del lagrimal hasta el final de la maxila superior; Inserción del pico (IP), desde el extremo del proceso supra-orbital lagrimal izquierdo al derecho; distancia entre narinas $(\mathrm{Nr})$, desde la zona interna de la cavidad narina izquierda a la derecha; distancia entre los pre-orbitales (PrO), desde el proceso orbital del lagrimal izquierdo al derecho; distancia inter-orbital (IO), desde el inter-orbital izquierdo al derecho; distancia entre los pos-orbitales (PsO), desde el proceso post-orbital izquierdo al derecho; inserción del atlas (IA), diámetro del foramen mágnum; ángulo del pico (AP), ángulo comprendido entre el angular de la mandíbula y el foramen mágnum (Tabla 1).

Tabla $N^{\circ}$ 1: Medidas de las variables morfométricas de los cráneos de algunas especies de picaflores de la Amazonia Peruana. Inserción del atlas (IA); distancia entre los pos-orbitales (PsO); distancia inter-orbital (IO); distancia entre los pre-orbitales (PrO); Inserción del pico (IP); distancia entre narinas (Nr); largo del pico (LP); ángulo del pico (AP).

\begin{tabular}{|c|c|c|c|c|c|c|c|c|}
\hline \multirow[b]{2}{*}{ Especies } & \multicolumn{8}{|c|}{ Medidas (mm) } \\
\hline & IA & PsO & IO & PrO & IP & $\mathbf{N r}$ & LP & $\mathbf{A P}$ \\
\hline $\begin{array}{l}\text { Thalurania furcata } \\
\text { (MZOr 00119) }\end{array}$ & 5.7 & 11.4 & 5.8 & 8.1 & 3.9 & 1.1 & 30.2 & 33 \\
\hline $\begin{array}{r}\text { Threnetes leucurus } \\
\text { (MZOr 00106) }\end{array}$ & 6.3 & 8.3 & 4.2 & 6.7 & 4.1 & 2 & 31.8 & 33 \\
\hline $\begin{array}{l}\text { Glaucis hirsuta } \\
\text { (MZOr 00108) }\end{array}$ & 4.7 & 8.8 & 4.3 & 7 & 4.5 & 2.5 & 29.5 & 34 \\
\hline $\begin{array}{c}\text { Phaethornisbourcieri } \\
\text { (MZOr 00140) }\end{array}$ & 5.2 & 8.6 & 4.7 & 7.1 & 3.2 & 1.5 & 30.4 & 37 \\
\hline $\begin{array}{l}\text { P. bourcieri } \\
\quad \text { (MZOr 00104) }\end{array}$ & 5.1 & 8.9 & 5.1 & 6.7 & 2.9 & 1.9 & 30.5 & 35 \\
\hline $\begin{array}{l}P \text {. superciliosus } \\
\text { (MZOr 00098) }\end{array}$ & 4.6 & 9.7 & 5.8 & 7.4 & 4.5 & 2.6 & 40.2 & 38 \\
\hline $\begin{array}{l}P \text { superciliosus } \\
\text { (MZOr 00122) }\end{array}$ & 4.5 & 10 & 5.9 & 7.9 & 4.2 & 2.4 & 39.8 & 38 \\
\hline $\begin{array}{l}P \text {. superciliosus } \\
\text { (MZOr 00123) }\end{array}$ & 4.4 & 9.9 & 5.3 & 7.6 & 4.5 & 2.6 & 39.8 & 38 \\
\hline $\begin{array}{l}\text { P. superciliosus } \\
\text { (MZOr 00130) }\end{array}$ & 4.8 & 9.7 & 5 & 7.5 & 4.3 & 2.2 & 40 & 35 \\
\hline $\begin{array}{l}\text { P. superciliosus } \\
\text { (MZOr 00137) }\end{array}$ & 4.4 & 9.5 & 4.9 & 7.4 & 4.3 & 2.2 & 41 & 39 \\
\hline $\begin{array}{l}\text { P. superciliosus } \\
\text { (MZOr 00139) }\end{array}$ & 4.3 & 9.8 & 5.3 & 7.7 & 4.4 & 2.6 & 39.7 & 41 \\
\hline
\end{tabular}


Para el análisis estadístico, con el fin de detectar patrones ecológicos y morfológicos entre las especies y las relaciones entre estos, se empleo el análisis de Componente Principal (CP). Este método es muy eficaz para el análisis de datos cuantitativos que se presentan bajo la forma de una matriz de $M$ observaciones / $N$ variables; esto nos permite visualizar y analizar rápidamente las correlaciones entre las $N$ variables y entre las $M$ observadas.

\section{RESULTADOS}

Las variables morfométricas no han mostrado correlación en algunas de ellas; indicándonos, así, que no son redundantes, ya que mostraron valores independientes una de las otras (Tabla 2). El análisis de los CP de las medidas morfométricas del cráneo de los colibríes nos dieron ocho CP con una acumulación de la varianza del $100 \%$, pero el CP1 y el CP2 muestran un porcentaje acumulado del $79 \%$, que son los componentes con mayor valor de variación. Así, el CP1 presento un valor propio de 4.305 con $53.811 \%$ de variabilidad y para el CP2 el valor propio fue de 2.021 con $25.263 \%$ de variabilidad.

El CP1 estuvo influenciado por las variables IA, Nr y AP. Dos de estas variables $(\mathrm{Nr}$ y $\mathrm{AP})$ mostraron estar correlacionadas negativamente con $r=$ -0.378 para $\mathrm{Nr}$ y $r=-0.387$ para AP. Ambas variables son ortogonalmente no correlacionadas con IA $(r=0.431)$ (Tabla 3). Para el caso del CP2, tres variables fueron las que influenciaron en su variación. Así, el PrO con una valor de correlación $(r)$ de 0.467 , la variable IO con $r=0.449$ y para $\mathrm{PsO}$ con $r=0.482$, estas variables se presentaron positivamente correlacionadas (Fig. 1).

Tabla 2. Matriz de correlación entre las variables morfométricas de los cráneos estudiados. Los valores en negrita son significativamente diferentes de 0 con un nivel de significación alfa $=0.05$

\begin{tabular}{crrrrrrrr}
\hline Variables & \multicolumn{1}{l}{ IA } & \multicolumn{1}{l}{$\mathrm{PsO}$} & \multicolumn{1}{l}{$\mathrm{IO}$} & \multicolumn{1}{l}{$\mathrm{PrO}$} & \multicolumn{1}{l}{$\mathrm{IP}$} & \multicolumn{1}{l}{$\mathrm{Nr}$} & \multicolumn{1}{l}{$\mathrm{LP}$} & \multicolumn{1}{l}{$\mathrm{AP}$} \\
\hline IA & $\mathbf{1}$ & -0.420 & -0.368 & -0.405 & $\mathbf{- 0 . 6 5 7}$ & $\mathbf{- 0 . 8 0 0}$ & -0.555 & $\mathbf{- 0 . 8 9 6}$ \\
$\mathrm{PsO}$ & -0.420 & $\mathbf{1}$ & $\mathbf{0 . 8 9 0}$ & $\mathbf{0 . 9 3 6}$ & 0.210 & 0.251 & 0.228 & 0.298 \\
$\mathrm{IO}$ & -0.368 & $\mathbf{0 . 8 9 0}$ & $\mathbf{1}$ & $\mathbf{0 . 7 6 8}$ & 0.111 & 0.268 & 0.281 & 0.368 \\
$\mathrm{PrO}$ & -0.405 & $\mathbf{0 . 9 3 6}$ & $\mathbf{0 . 7 6 8}$ & $\mathbf{1}$ & 0.192 & 0.196 & 0.201 & 0.333 \\
$\mathrm{IP}$ & $\mathbf{- 0 . 6 5 7}$ & 0.210 & 0.111 & 0.192 & $\mathbf{1}$ & $\mathbf{0 . 8 9 8}$ & 0.373 & 0.403 \\
$\mathrm{Nr}$ & $\mathbf{- 0 . 8 0 0}$ & 0.251 & 0.268 & 0.196 & $\mathbf{0 . 8 9 8}$ & $\mathbf{1}$ & 0.456 & $\mathbf{0 . 6 2 2}$ \\
$\mathrm{LP}$ & -0.555 & 0.228 & 0.281 & 0.201 & 0.373 & 0.456 & $\mathbf{1}$ & $\mathbf{0 . 6 4 7}$ \\
$\mathrm{AP}$ & $\mathbf{- 0 . 8 9 6}$ & 0.298 & 0.368 & 0.333 & 0.403 & $\mathbf{0 . 6 2 2}$ & $\mathbf{0 . 6 4 7}$ & $\mathbf{1}$ \\
\hline
\end{tabular}


Tabla $N^{\circ}$ 3: Valores propios de los dos componentes principales (PC) y valores de correlación de las medidas morfológicas de los cráneos de las especies de colibríes estudiadas (ver texto).

\begin{tabular}{|c|c|c|}
\hline & $\mathrm{PC} 1$ & $\mathrm{PC} 2$ \\
\hline Valor propio & 4.305 & 2.021 \\
\hline Variabilidad (\%) & 53.811 & 25.263 \\
\hline$\%$ acumulado & 53.811 & 79.074 \\
\hline \multicolumn{3}{|l|}{ Variables } \\
\hline IA & 0.431 & 0.210 \\
\hline $\mathrm{PsO}$ & -0.339 & 0.482 \\
\hline $\mathrm{IO}$ & -0.325 & 0.449 \\
\hline $\mathrm{PrO}$ & -0.323 & 0.467 \\
\hline IP & -0.321 & -0.342 \\
\hline $\mathrm{Nr}$ & -0.378 & -0.339 \\
\hline LP & -0.307 & -0.188 \\
\hline $\mathrm{AP}$ & -0.387 & -0.191 \\
\hline \multicolumn{3}{|l|}{ Observación * } \\
\hline MZOr 00119 & 0.684 & 4.081 \\
\hline MZOr 00106 & 3.433 & -1.185 \\
\hline MZOr 00108 & 1.125 & -1.667 \\
\hline MZOr 00140 & 2.355 & 0.145 \\
\hline MZOr 00104 & 2.384 & 0.242 \\
\hline MZOr 00098 & -1.882 & -0.318 \\
\hline MZOr 00122 & -2.134 & 0.799 \\
\hline MZOr 00123 & -1.938 & -0.448 \\
\hline MZOr 00130 & -0.416 & -0.132 \\
\hline MZOr 00137 & -1.172 & -0.924 \\
\hline MZOr 00139 & -2.438 & -0.594 \\
\hline
\end{tabular}

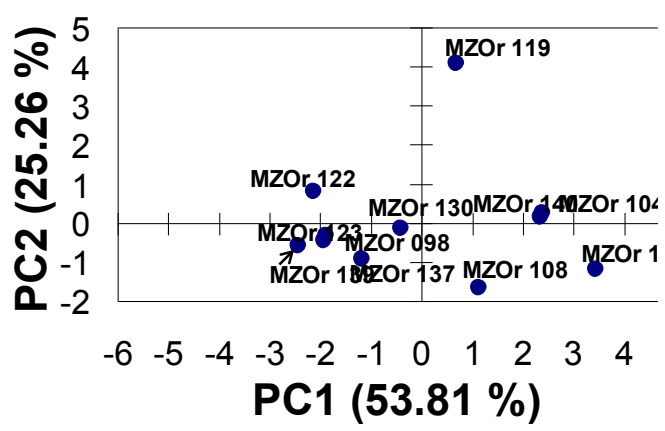

Fig. 1

\section{DISCUSIÓN}

La tendencia de los Phaethornis bourcieri fue la visita a flores con corolas cortas y flores con curvaturas mayores que la curvatura del pico (Rodríguez y Gary, 2003). Esta tendencia esta también influenciada por los caracteres de la inserción del pico. Lo que le permite visitar flores pequeñas. Sin embargo, para Phaethornis superciliosus, los caracteres orbítales juega un papel importante en la selección de la flor, ya que, estos caracteres están correlacionados para sostener un pico largo y curvo lo que le puede permitir vistas a diferentes tipos de flores, como ocurre con Phaethornis malaris, que tiene parecida estructura de pico y que visita gran número de flores de diferentes formas (Rodríguez y Gary, op cit).

Threnetes leucurus, al igual que Phaethornis bourcieri, tienen la mor- 
fología del pico similar y por lo tanto tienden a visitar un espectro corto de flores. El carácter de la inserción del pico y los caracteres orbítales, en Threnetes leucurus, no tiene gran relevancia para la especie, pero, para Thalurania furcata el carácter de los orbitales influyo mucho en el tamaño del pico lo que podemos predecir que la selección de flores podría ser restringida como ocurre con Threnetes leucurus y Phaethornis bourcieri. Entonces, Altshuler \& Clark (2003) sugieren que las formas adaptativas del pico de los picaflores esta coevolutivamente relacionadas con las formas de las flores, pero que esta adaptación no es estrictamente especifica porque puede existir que el ave cambie de flor según las necesidades de energía nectarea que encuentran.

Dentro de los colibríes existieron tendencias morfométricas en la inserción del atlas (IA) y en el ángulo de inserción del pico (AP), los cuales se relacionan con la longitud y curvatura del pico (Rodríguez y Gary 2005). Estas características morfométricas predominaron en la mayoría de los cráneos estudiados, entendiéndose pues que las especies de colibríes estudiadas comparten aspectos ecológicos similares, tales como las flores que visitan.

\section{CONCLUSIONES}

Los picos de las especies Thalurania furcata, Threnetes leucurus, Glaucis hirsuta y Phaethornis bourcieri están fuertemente asociados a la Inserción del atlas (IA) y los picos de Phaethornis superciliosus por la distancia entre las Narinas (Nr) y el ángulo de inserción del pico con respecto a la columna (AP).

Las variables morfométricas de los cráneos, en las especies de colibríes, comparten estrecha relación con la ecología del ave.

\section{LITERATURA CITADA}

ALTSHULER, L. D, C. J, CLARK. 2003. Darwin's hummingbirds. Science 300: 588-589.

BROWN, J.H., M. A. BOWERS. 1985. Community organization in hummingbirds: relationships between morphology and ecology. The Auk 102: 251-2969.

COTTON, P. 1998. Coevolution in Amazonian hummingbird-plant community. Ibis 140: 639-646.

HANKEN, J, B. K. HALL. 1993. The Skull: Patterns of Structural and Systematic Diversity.Págs.391-437. RODRÍGUEZ. F. C, F. GARY. 2005. Análisis ecomorfológico de una comunidad de colibríes ermitaños (Trochilidae, Phaethornidae) y sus 
flores en la Amazonia Colombiana.

Ornitología Colombiana 3: 7-27.

TEMELES, E. J, W.J. KRESS. 2003.

Adaptation in a planthum-

mingbird association. Science 300: 630-633.

TEMELES, E. J, LINHART. Y. B, M. MASONJONES, H. D. MASONJONES. 2002. The Role of Flower Width in Hummingbird Bill Length-Flower Length Relationship. Biotropica 34 (1): 68-80.

\section{AGRADECIMIENTOS}

A Mercedes Gonzáles, directora del Museo de Historia Natural de la Universidad Ricardo Palma, por permitirme el uso de las colecciones de cráneos de aves (Fam. Trochilidae). A Víctor R. Morales por su motivación y constante orientación durante la realización de la presente investigación. 